\title{
HUGO GYLDÉN. \\ Ein biographischer Umriss nebst einigen Bemerkungen über seine wissenschaftlichen Methoden \\ VON
}

KARL BOHLIN.

Johan August Hugo Grudén wurde am 29 Mai I 84 I in Helsingfors geboren. Sein Vater war der Professor der griechischen Sprache an der k. k. Alexanderuniversität, Nirs Abraham Gysdén. Die Mutter besass eine den damaligen Standpunkt der Frauenerziehung weit überragende Bildung und sogar eine ungewöhnliche Gelehrsamkeit. So wurde es Gyldén bescheert im elterlichen Hause eine umsichtsvolle und gründliche Unterricht zu geniessen. Er kam darauf in eine private Schule und wurde mit sechzehn Jahren I 857 als Student an der Universität eingeschrieben. Er widmete sich als solcher anfangs zwei Jahre der Chemie, wandte sich aber dann den astronomisch-mathematischen Disciplinen zu. Schon im Jahre I 860 promovirte er zum Phys. Math. Magister und zog darauf zur weiteren Ausbildung ins Ausland.

Die Reise ging nach Deutschland und zwar hielt er sich in den Jahren I 86I-62 vornehmlich bei Hansen, dem Altmeister der theoretischen Astronomie, in Gotha auf. Nachdem er noch Leipzig besucht hatte, kehrte er nach der Heimath zurück, um seine akademischen Studien zu beendigen. Inzwischen war eine Lehrerstelle für Astronomie an der Universität in Helsingfors frei geworden und Gyldén sandte auch aus Gotha, als er davon Nachricht bekommen hatte, an die Behörden ein Gesuch ein, das indessen zu spät ankam und desswegen vereitelt wurde. Nach der Rück-

Aota mathomatioa. 20. Imprime le 28 octobre 1897. 
Karl Bohlin.

kehr nach Helsingfors legte er I 862 das Licentiatexamen ab, wurde im December desselben Jahres zum Docenten der Astronomie und im folgenden Jahre zum Doctor der Philosophie ernannt.

Schon ehedem hatte Gyldén eine vorläufige Anstellung bei der Pulkowaer Sternwarte angenommen und wurde nun daselbst I 863 zum Adjunctastronomen und I 865 zum »älteren Astronomen» befördert. In Pulkowa entwickelte er als beobachtender Astronom eine aussergewöhnlich rege Thätigkeit, deren die Annalen der Pulkowaer Sternwarte Zeugniss ablegen, fand aber trotzdem die Musse, sich theoretischen Untersuchungen zu widmen. Seine Untersuchungen über die Constitution der Atmosphäre und die Strahlenbrechung in derselben wie die Abhandlung Studien auf dem Gebiete der Störungstheorie stammen aus dieser Zeit.

Einen bedeutungsvollen Wendpunkt seiner wissenschaftlichen Laufbahn brachte das Jahr I871. Der Director der Sternwarte in Stockholm Nils Haqurn Selander war gestorben und die Wahl der Academie der Wissenschaften fiel auf Gyldén als dessen Nachfolger. Er bekleidete dieses Amt als Astronom der Academie der Wissenschaften und Director der Sternwarte von I 87 I an bis zu seinem Tode. Auf diesem Posten wurde es ihm ermöglicht, eine für die Astronomie sehr fruchtbare Wirksamkeit zu entfalten. Nicht nur dass seine Studien und Untersuchungen auf dem Gebiete der Störungstheorie und über verwandte Gegenstände in einer überaus lebhaften schriftstellerischen Thätigkeit sich entwickelten und mit der Zeit allmählig den Character eines definitiv ausgearbeiteten Systemes annahmen: er verstand es auch eine Reihe von Schülern, hauptsächlich aus Russland und Deutschland, heranzuziehen, die kürzere oder längere Zeit sich bei ihm über die Theorien der intermediären und absoluten Bahnen informirten und durch selbständige Arbeiten nicht unbedeutend zur Verbreitung der Kenntniss und zur Ausbildung jener Theorien beigetragen haben. Ein Theil dieser Untersuchungen sind in der von Gyldén gegründeten Publication Astronomiska iakttagelser och undersökningar anstälda på Stockholms observatorium veröffentlicht worden.

Als die theoretischen Grundlagen der intermediären und absoluten Bahnen der Himmelskörper, worüber Gyldén zum ersten Mal in drei in schwedischer Sprache geschriebenen Abhandlungen, Undersökningar af teorien för himlakropparnes rörelser, I-III, 1 $88 \mathrm{I}-82$, berichtete, soweit gediehen waren, dass zur numerischen Verwerthung derselben geschritten werden 
konnte, bewilligte der schwedische Reichstag im Jahre I 884 eine Summe von I 2,000 Kronen zur Ausführung von numerischen Rechnungen betreffend die absoluten Bahnen der grossen Planeten. Diese Rechnungen wurden auch sofort in Angriff genommen und kräftig gefördert. Für die meisten Combinationen der grossen Planeten wurden so die grundlegenden Rechnungen - Entwicklung der Störungsfunction und Integration der gewöhnlichen Störungsglieder — fertig gestellt. Ein, wenn auch nur sehr kleiner Theil der Resultate sind in dem nach seinem Tode erschienenen unvollendeten zweiten Theile seines Werkes Traité analytique des orbites absolues des huit planètes principales publicirt worden. ${ }^{1}$

Schon seit vielen Jahren hatte Gyldén an einem Herzleiden gelitten. Im vorigen Sommer nahm die Krankheit einen drohenden Character, schien sodann nach emem Aufenthalte in Nauheim etwas nachzugeben, aber nahm im Herbste wieder heftig zu. Am 9 November des vergangenen Jahres entriss ihn mitten im Schaffen der Tod seiner Familie und der Wissenschaft.

Gyldén war Mitglied der schwedischen Academie der Wissenschaften, der Wissenschaftssocietät in Upsala, der Wissenschaftssocietät in Helsingfors, der französischen Academie als Nachfolger Secchi's, der russischen Academie der Wissenschaften, der Royal Astronomical Society und anderer gelehrten Gesellschaften. Von I 889 bis 1896 fungirte er als Vorsitzender der Astronomischen Gesellschaft. Im Jahre I 879 wurde ihm eine Professorsstelle in seiner Vaterstadt und zwei mal wurden ihm im Auslande Professorsstellen angeboten, einmal i 876 in Gotha, das andere Mal I884 in Göttingen. Bei dieser letzten Gelegenheit hatte er auch eine Übersiedlung auf deutschen Boden ernstlich erwogen, liess sich aber auf den besonderen Wunsch der Academie der Wissenschaften hin bewegen, seine Absicht aufzugeben. Seinem Wunsch durch Vorlesungen zu wirken wurde bei dieser Gelegenheit durch besonders für den Zweck zur Disposition gestellten Mittel entsprochen. Von 1888 an war er als Lehrer der Astronomie bei der Hochschule in Stockholm thätig. Seit der Gründung dieser Zeitschrift gehörte er ihrer Redaction.

Der mathematische Inhalt der Publicationen Gyldéns ist mit seinen astronomischen Untersuchungen so nahe verbunden, dass es schwer fällt, die mathematischen Resultate von den astronomischen Aufgaben, deren

1 Die Fortsetzung dieser Untersuchungen ist von Herrn Director O. BAckLund übernommen worden. 
Lösung er nachstrebte, zu trennen. Ohne Zweifel würde es leichter sein, eine Vorstellung der durch ihn erlangten astronomischen Resultate auf dem Gebiete der Störungen zu bilden, ohne die mathematischen Methoden zur Hülfe zu nehmen, deren er sich bediente. Die gewonnenen Resultate dürften nämlich durch allgemeinere functionstheoretische Methoden und auf anderen Wegen, als die elliptischen Functionen ihm eröffneten, wiedergefunden werden können. Allerdings haben sich die elliptischen Functionen in seinen Händen als sehr fruchtbar erwiesen, indem nämlich die genau studirten Eigenschaften, die diese Functionen über die trigonometrischen hinaus besitzen, bei der Anwendung auf die Astronomie unmittelbar verwerthet werden konnten. Ein weiterer Vortheil der Anwendung dieser Functionen lag darin, dass die von Herrn Hermite behandelte s. g. LAMÉ-sche Differentialgleichung benutzt werden konnte, um eine erste Verbesserung eines durch einfache Verbindungen elliptischer Functionen dargestellten preliminären Resultates abzuleiten. Wollte man aber die Annäherungen über diesen Genauigkeitsgrad fortsetzen, so wäre man in der Regel zu elementären Hülfsmitteln zurückgewiesen. Die elliptische Functionen sind in dem Sinne ein Mittel, durch welches es ermöglicht wird, zu einem gewissen Grade in das Störungsproblem einzudringen, eignen sich aber weniger um dasselbe nach einem einheitlichen Algoritmus endgültig zu behandeln. Diesem Umstande sind die Schwierigkeiten zuzuschreiben, welche Gyldéns Methoden darbieten. In dieser Zeitschrift hat Gyldén seine Untersuchungen über das allgemeine Problem der Störungen mit besonderer Rücksicht auf die Convergenz der Reihen in zwei grösseren Abhandlungen: Untersuchungen über die Convergenz der Reihen, welche zur Darstellung der Coordinaten der Planeten angewendet werden, Acta mathematica 9, 1887 und Nouvelles recherches sur les séries employées dans les théories des planètes, Acta mathematica 15, 1891; 17, 1892, veröffentlicht. Dieselben sind in der Vierteljahrsschrift der Astronomischen Gesellschaft für I 893 umständlich analysirt worden. In der ersten dieser Abhandlungen wird die Ermittlung der sog. characteristischen Glieder auf eine Reihe ähnlicher Operationen durch elliptische Functionen in überraschend eleganter Weise zurückgeführt. Der interessanteste Gegenstand der zweiten Abhandlung, nämlich der Versuch, die Convergenz der Reihen im Anschluss an gewissen höheren Potenzen der Unbekannten mittels sog. horistischer Glieder; welche durch die Transformationen in den Differen- 
tialgleichungen entstehen, festzustellen, entbehrt hier die Klarheit einer endgültigen Darstellung und beschäftigte Gyldén noch immer in der letzten Zeit.

Abgesehen von diesen Convergenzfragen besitzen die astronomischen Methoden Gyldéns einen unstreitbaren Vorzug vor anderen, älteren $\mathrm{Me}$ thoden. Vom Anfange seiner Untersuchungen über die allgemeinen Störungen an war es nämlich sein Streben, dieselben in rein periodischer Form darzustellen. Die in einer solchen analytischen Form dargestellte Bahn eines Himmelskörpers nannte er eine absolute, weil dieselbe für alle Zeiten von der wirklichen Bahn nur um kleine periodische Grössen von der Ordnung der Masse sich unterscheidet. Die wirkliche Berechnung solcher Bahnen führt $\mathrm{zu}$ einer gewissen Wahl von Integrationsconstanten, für welche Gyldén auch eine besondere Terminologie aufgestellt hat. Diese Theorie der absoluten Bahnen entspricht nach den älteren Störungstheorien den s. g. sacularen Störungen. Seine umfassenden Untersuchungen über diesen Gegenstand hat Gyldén im ersten Theile seines unvollendeten Werkes: Traité analytique des orbites absolues des huit planètes principales, Stockholm I 893, nachgelassen.

Aber nicht nur für die Theorie der allgemeinen Störungen bieten die elliptischen Functionen eine vortheilhafte Anwendung, indem man gewisse Glieder aus der Störungsfunction mit den Differentialgleichungen schon bei der ersten Näherung vereinigt, wodurch diese, die ja ohne jene Glieder zu Ausdrücken rein trigonometrischer Art führen, durch elliptische Functionen integrierbar werden. Für gewisse Fälle, besonders bei der numerischen Berechnung sehr excentrischer Bahnen wie derjenigen der Cometen, hat HANSEN einen praktisch sehr schätzbaren Kunstgriff eingeführt, ohne welchen sogar eine analytische Behandlung solcher Fälle scheitert. Der Grundgedanke des Verfahrens ist folgender. Die Bahn des Himmelskörpers wird in zwei oder mehreren Theilen zerlegt und für jeden Theil drückt man die Coordinaten durch ein besonderes Hülfsargument aus. Dieses Argument - die sog. partielle Anomalie - wird in einer solchen Weise gewählt, dass dasselbe von $0^{\circ}$ bis $180^{\circ}$ variirt, während der Himmelskörper den betreffenden Theil der Bahn beschreibt. Ausserhalb der Grenzen dieses Theilgebietes verlieren die nach der partiellen Anomalie entwickelten Reihen ihre Gültigkeit. Wie Gyldén zuerst gezeigt hat, bieten sich die elliptischen Functionen fast von selbst zur Einführung solcher partieller Anomalien und 
sind ausserdem wegen der raschen Convergenz der Reihenentwicklungen für diesen Zweck sehr geeignet. Gyldéns erste grössere theoretische Arbeit, Studien auf dem Gebiete der Störungstheorie, I, Petersburg 1871, behandelt die Aufgabe, besondere für den genannten Zweck nutzbare Entwicklungen der elliptischen Functionen herzustellen. Ein Theil der betreffenden Resultate waren wohl schon von C. O. MeIEn im 37 Bande von Crelles Journal für reine und angewandte Mathematik angegeben. Weil sie aber weder für die praktische Benutzung gestellt oder hinreichend vollständig waren, entschloss sich Gyldén, eine neue Behandlung der Frage im Anschluss an die Fundamenta $\mathrm{J}_{\mathrm{ACOBI}}$ 's vorzunehmen. Diese Abhandlung enthält zuerst trigonometrische Entwicklungen von Potenzen der elliptischen Functionen wie $\left(\sin \operatorname{am} \frac{2 K}{\pi} x\right)^{2 n},\left(\sin \operatorname{am} \frac{2 K}{\pi} x\right)^{2 n+1}$ u. s. w. für $n=0$, I , 2 . Von besonderem Interesse sind die darauf folgenden Entwicklungen für grosse $n$-Werthe von gewissen elliptischen Integralen, wie z. B.

$$
\begin{gathered}
\int_{0}^{1} \frac{x^{2 n} d x}{\sqrt{\left(1-x^{2}\right)\left(\mathrm{I}-k^{2} x^{2}\right)}} \\
=\frac{\pi}{2 k^{\prime}} \frac{1 \cdot 3 \ldots 2 n-1}{2 \cdot 4 \ldots 2 n}\left\{1-\frac{1}{2} \frac{\mathrm{I}}{2 n+2} \frac{k^{2}}{k^{\prime 3}}+\frac{\mathrm{I} \cdot 3}{2 \cdot 4} \frac{\mathrm{I} \cdot 3}{(2 n+2)(2 n+4)}\left(\frac{k^{2}}{k^{\prime 2}}\right)^{2} \ldots\right\} .
\end{gathered}
$$

Diese Reihenentwicklungen eignen sich für grosse Werthe von $n$ zur numerischen Rechnung, sind aber für $\frac{k}{k^{\prime}}>\mathrm{I}$, also für den Fall, der bei Cometenbahnen vorzugsweise vorkommt, halbconvergent. Hierauf folgen Entwicklungen von Potenzen gewisser Producte elliptischer Functionen und zum Schluss Entwicklungen bei dem Auftreten von Vielfachen einer Amplitudo wie z. B. die Bestimmung der Coëfficienten der Reihe

$$
\sin \left(n \operatorname{am} \frac{2 K}{\pi} x\right)=2 \sum_{1}^{n} \sin x+2 \sum_{2}^{n} \sin 2 x+\ldots
$$

Als eine Eigenthümlichkeit für die Deduction dieser Resultate kann erwähnt werden, dass der Verfasser sich des Fourier'schen Theoremes nicht bedient, sondern gewisse dreigliedrige Recursionsformeln zwischen den Coëfficienten aufstellt, die dann, als Differenzgleichungen betrachtet, nach der IAAPLACE'schen Methode integrirt werden. 
Zu den interessantesten Arbeiten Gyldéns gehören zwei Abhandlungen, die mit der Frage von partiellen Anomalien nahe zusammenhängen, nämlich Om summationen af periodiska serier, Stockholm 1872, und Sur la sommation des fonctions périodiques, Annales de l'école normale i 879. Eins der Hauptresultate dieser Abhandlungen ist eine Generalisation der sog. Mac Laurin'schen Formel

$$
z_{s}=\frac{1}{\pi} \operatorname{Lim}_{k=\infty} \int_{0}^{s \pi} F(t) \frac{\sin (2 t+1) t}{\sin t} d t
$$

zu einer Relation von der Form

$$
z_{s}=\frac{1}{2} \int_{0}^{s \pi}\left\{F(t)+b_{1}^{(i)} \frac{d^{2} F(t)}{d t^{2}}+\ldots+b_{i}^{(i)} \frac{d^{s i} F(t)}{d t^{2 i}}\right\} \chi_{i}(t) d t
$$

Im Zusammenhange mit dieser Formel steht die bemerkenswerthe Entwicklung eines Bogens $\vartheta$ in die periodische Reihe

$$
\vartheta=\sum_{1}^{\infty} \beta_{2 n}^{(i)} \sin 2 n \vartheta+\frac{\pi}{2} \sum_{0}^{i} \alpha_{2 n+1}^{(i)} \sin (2 n+1) \vartheta \quad\left(-\frac{\pi}{2}<\vartheta<\frac{\pi}{2}\right)
$$

so auch ganz ähnliche Reihenentwicklungen für $\sin \mu \vartheta$, $\cos \mu \vartheta$, wo $\mu$ eine irrationale Zahl bedeutet. Diese Formeln ergeben sich, wenn man sich die Aufgabe stellt, eine Entwicklung von $(\cos \vartheta)^{2 i+1}$ nach geraden Vielfachen von $\vartheta$

$$
C_{0}^{(i)}-{ }_{2} C_{2}^{(i)} \cos 2 \vartheta-{ }_{4} C_{4}^{(i)} \cos 4 \vartheta-\ldots
$$

zu finden, und wurden schon 1867 von Gyldén in seiner Abhandlung Relationer mellan cosiner och siner för irrationella vinklar, Helsingfors i 867, gegeben. Mit diesen Reihenentwicklungen aufs engste verbunden ist die Theorie für den sog. separirenden Factor — eine periodische Reihe der oben erwähnten Art, die innerhalb der angegebenen Grenzen einen constanten Werth z. B. die Einheit darstellt, ausserhalb derselben aber eine gewissermassen beliebige Werthfolge representirt.

Von späteren Untersuchungen auf dem rein mathematischen Gebiete legte Gyldén selbst auf dem kleinen Aufsatze Om sannolikheten att päträffa stora tal vid utvecklingen af irrationela decimalbrák $i$ kedjebråk, Stockholm I 888, einen besonderen Werth. 
Karl Bohlin.

Die Zahl der Publicationen Gyldéns beläuft sich auf nicht viel weniger als zwei hundert. Für das Verzeichniss über dieselben gestatten wir uns auf eine demnächst in der Vierteljahrsschrift der Astronomischen Gesellschaft erscheinende Biographie hinzuweisen. Das meiste dieser grossen schriftstellerischen Wirksamkeit bezieht sich auf astronomische Fragen. Aber ausser der verschiedensten Gegenständen der theoretischen und praktischen Astronomie, die in dieser langen Reihe von grösseren und kleineren Abhandlungen und Zeitschriftpublicationen vertreten sind, finden wir auch eine Anzahl Aufsätze über Versicherungswesen sowie Vorträge und Mittheilungen in philosophischen und socialen Fragen. 